\title{
Thermal buckling analysis of ceramic-metal functionally graded plates
}

\author{
Ashraf M. Zenkour ${ }^{1,2 *}$, Daoud S. Mashat ${ }^{1}$ \\ ${ }^{1}$ Department of Mathematics, Faculty of Science, King AbdulAziz University, Jeddah, Saudi Arabia; \\ *Corresponding Author: zenkour@kau.edu.sa \\ ${ }^{2}$ Department of Mathematics, Faculty of Science, Kafrelsheikh University, Kafr El-Sheikh, Egypt
}

Received 7 March 2010; revised 20 April 2010; accepted 26 April 2010.

\begin{abstract}
Thermal buckling response of functionally graded plates is presented in this paper using sinusoidal shear deformation plate theory (SPT). The material properties of the plate are assumed to vary according to a power law form in the thickness direction. Equilibrium and stability equations are derived based on the SPT. The non-linear governing equations are solved for plates subjected to simply supported boundary conditions. The buckling analysis of a functionally graded plate under various types of thermal loads is carried out. The influences of many plate parameters on buckling temperature difference will be investigated. Numerical results are presented for the SPT, demonstrating its importance and accuracy in comparison to other theories.
\end{abstract}

Keywords: Thermal Buckling; Non-Linear Strains; Functionally Graded Material; Sinusoidal Plate Theory; Thermal Load

\section{INTRODUCTION}

The rapid development of composite materials and structures in recent years has drawn increased attention from many engineers and researchers. These materials are broadly used in aerospace, mechanical, nuclear, marine, and structural engineering. In conventional laminated composite structures, homogeneous elastic laminas are bonded together to obtain enhanced mechanical and thermal properties. However, the abrupt change in material properties across the interface between different materials can result in large inter-laminar stresses leading to delimitation, cracking, and other damage mechanisms which result from the abrupt change of the mechanical properties at the interface between the layers. To remedy such defects, functionally graded materials (FGMs), within which material properties vary continuously, have been proposed.

The concept of FGM was proposed in 1984 by a group of materials scientists, in Sendai, Japan, for thermal barriers or heat shielding properties. Initially FGM was designed as a thermal barrier material for aerospace application and fusion reactors. Later on FGM was developed for the military, automotive, biomedical and semiconductor industries, and as a general structural element in high thermal environments. FGM is one of the advanced high temperature materials capable of withstanding extreme temperature environments. FGMs are composite and microscopically heterogeneous in which the mechanical properties vary smoothly and continuously from one surface to the other. This is achieved by gradually varying the volume fraction of the constituent materials. Typically, these materials are made from a mixture of ceramics and metal or a combination of different materials. The ceramic constituent of the material provides the high-temperature resistance due to its low thermal conductivity and protects the metal from oxidation. The ductile metal constituent, on the other hand, prevents fracture caused by stresses due to high-temperature gradient in a very short period of time. Further, a mixture of a ceramic and a metal with a continuously varying volume fraction can be easily manufactured [1-4].

A comprehensive work on the FGMs was presented in the literature. The response of FG ceramic-metal plates has been investigated by Praveen and Reddy [5] using a plate finite element. They investigated the static and dynamic thermoelastic responses of the FGMs by varying the volume fraction using a simple power law distribution. Reddy [6] developed the Navier's solutions for FG plates using the third-order shear deformation plate theory (TSDT) and an associated finite element model. Amini et al. [7] described a method for three-dimensional free vibration analysis of rectangular FGM plates 
resting on an elastic foundation using Chebyshev polynomials and Ritz's method. This analysis has been based on a linear, small-strain, three-dimensional elasticity theory. Analysis of FG plates under static and dynamic loads has been presented by Sladek et al. [8] using the meshless local Petrov-Galerkin method and ReissnerMindlin theory to describe the plate bending problem. Kim et al. [9] investigated finite element computation of fracture parameters in FGM assemblages of arbitrary geometry with stationary cracks. In Altenbach and Eremeyev [10], a viscoelastic FG polymer foam has been studied using a new plate theory based on the direct approach. The large deflection response of simply supported rectangular FG plates under normal pressure loading has been analyzed by Ovesy and Ghannadpour [11] using a finite strip method. In Han [12], a numerical method was proposed for analyzing transient waves in plates of FGM excited by impact loads. The bending problem of transverse load acting on FGM rectangular plate using both two-dimensional trigonometric and three-dimensional elasticity solutions was investigated by Zenkour [13]. Zenkour [14,15] studied the bending response, buckling and free vibration of simply supported FG sandwich plate using the SPT. Zenkour [16] presented the derivation of equations for free vibration of FG plates expressing the displacement components by trigonometric series representation through the plate thickness. Other researches into FGMs have included the nonlinear analysis of FG plates [17], large deformation analysis of FG shells [18], static and vibration analysis of FG beams [19,20].

In view of the advantages of FGMs, a number of investigations dealing with thermal buckling had been published in the scientific literature. In recent years, the mechanical and thermal buckling analysis of FG ceramic-metal plates has been presented by Zhao et al. [21] using the first-order shear deformation plate theory, in conjunction with the Ritz method. A two-dimensional global higher-order deformation theory has been employed by Matsunaga [22] for thermal buckling of plates made of FGMs. Morimoto et al. [23] presented the thermal buckling analysis of FG rectangular plates subjected to partial heating in a plane and uniform temperature rise through its thickness. In Ref. [24], Shariat and Eslami presented the thermal buckling analysis of rectangular FG plates with geometrical imperfections using the classical plate theory to derive the equilibrium, stability, and compatibility equations of an imperfect FGM. Thermal buckling of rectangular and circular plates compose of FGM was also studied based on the first- and higher-order shear deformation plate theory [25-27].

Various plate theories, depending upon the throughthickness displacement pattern considered, have been used to determine thermal buckling loads of composite plates. The classical plate theory [24], which is based on Kirchhoff's hypothesis, overestimates the thermal buckling load when applied to even moderately thick plates. This is particularly true for composite plates in which transverse shear moduli are small in comparison to the in-plane Young's moduli [28]. In such cases, it becomes necessary to take into account shear deformation effects. Thus, various improved plate theories such as first-order shear deformation [25,26], higher order shear deformation [5,6] and sinusoidal shear deformation [13-16,2931] plate theories have been developed to predict the behavior of plates with thickness shear deformation. In this article, thermal buckling analysis of rectangular FG ceramic-metal plates is investigated. The material properties of the FG plates are assumed to vary continuously through the thickness, according to a simple power law distribution of the volume fraction of the constituents. The SPT is used to obtain the buckling of the plate under different types of thermal loads. The thermal loads are assumed to be uniform, linear and non-linear distribution through the thickness. Additional numerical results are presented for FGM plates that show the effects of various parameters on thermal buckling response.

\section{MATHEMATICAL MODEL}

Consider a rectangular plate of length $a$, width $b$ and thickness $h$ made of FGM. The plate is subjected to a thermal load $T(x, y, z)$. The material properties of the FGM plate, such as Young's modulus $E$ and thermal expansion coefficients $\alpha$ are assumed to be functions of the volume fraction of the constituent materials. The FGM plate is supported at four edges defined in the $(x, y, z)$ coordinate system with $x$ - and $y$-axes located in the middle plane $(z=0)$ and its origin placed at the corner of the plate.

The modulus of elasticity $E$, the coefficient of thermal expansion $\alpha$ and Poisson's ratio $v$ are assumed as [5]

$$
\begin{aligned}
& E(z)=E_{\mathrm{m}}+E_{\mathrm{cm}} V^{k}, \\
& \alpha(z)=\alpha_{\mathrm{m}}+\alpha_{\mathrm{cm}} V^{k}, v(z)=v_{0},
\end{aligned}
$$

where

$$
\begin{aligned}
& E_{\mathrm{cm}}=E_{\mathrm{c}}-E_{\mathrm{m}}, \\
& \alpha_{\mathrm{cm}}=\alpha_{\mathrm{c}}-\alpha_{\mathrm{m}}, \\
& V=\left(\frac{z}{h}+\frac{1}{2}\right),
\end{aligned}
$$

and $E_{\mathrm{m}}$ and $\alpha_{\mathrm{m}}$ denote the elastic moduli and the coefficient of thermal expansion of metal; $E_{\mathrm{c}}$ and $\alpha_{\mathrm{c}}$ denote the elastic moduli and the coefficient of thermal 
expansion of ceramic, and $k$ is the volume fraction exponent. The value of $k$ equal to zero represents a fully ceramic plate. The above power law assumption reflects a simple rule of mixtures used to obtain the effective properties of the ceramic-metal plate. The rule of mixtures applies only to the thickness direction. The density of the plate varies according to the power law, and the power law exponent may be varied to obtain different distributions of the component materials through the thickness of plate. Note that the volume fraction of the metal is high near the bottom surface of the plate, and that of ceramic high near the top surface. In addition, Eq. 1 indicates that the bottom surface of the plate $(z=-h / 2)$ is metal-rich whereas the top surface $(z=h / 2)$ of the plate is ceramic-rich. For simplicity, $v$ is assumed constant across the plate thickness.

The displacements of a material point located at $(x, y$, $z$ ) in the FGM plate might better be illustrated as [29, 30]:

$$
\left.\begin{array}{l}
u_{1}(x, y, z)=u-z \frac{\partial w}{\partial x}+\Psi(z) \varphi_{1}, \\
u_{2}(x, y, z)=v-z \frac{\partial w}{\partial y}+\Psi(z) \varphi_{2}, \\
u_{3}(x, y, z)=w
\end{array}\right\}
$$

where $u, v$ and $w$ are the displacements of the middle surface along the axes $x, y$ and $z$, respectively, and $\varphi_{1}$ and $\varphi_{2}$ are the rotations about the $y$ and $x$-axes and account for the effect of transverse shear. The coefficient of $\varphi_{1}$ or $\varphi_{2}$ which is given by $\Psi(z)$ should be odd function of $z$. All of the generalized displacements $\left(u, v, w, \varphi_{1}, \varphi_{2}\right)$ are functions of the $(x, y)$. The displacement of the classical thin plate theory (CPT) can easily be obtained by setting $\Psi(z)=0$. The displacements of the first-order shear deformation plate theory (FPT) are obtained by setting $\Psi(z)=z$. In addition, the higher-order shear deformation plate theory (HPT) [6] is obtained by setting

$$
\Psi(z)=z\left[1-\frac{4}{3}\left(\frac{z}{h}\right)^{2}\right] .
$$

Also, the SPT is obtained by setting $[14,15]$ :

$$
\Psi(z)=\frac{h}{\pi} \sin \left(\frac{\pi z}{h}\right) .
$$

Note that the present SPT, as well as HPT, is simplified by enforcing traction-free boundary conditions at the plate faces. The SPT accounts according to a cosine-law distribution of the transverse shear deformation through the thickness of the FGM plate. The SPT, HPT and FPT contain the same number of dependent un- knowns. No transversal shear correction factors are needed for both SPT and HPT because a correct representation of the transversal shearing strain is given.

The non-linear strain components $\varepsilon_{i j}$ compatible with the displacement field in Eq.3 are

$$
\begin{gathered}
\left\{\begin{array}{l}
\varepsilon_{11} \\
\varepsilon_{22} \\
\varepsilon_{12}
\end{array}\right\}=\left\{\begin{array}{l}
\varepsilon_{11}^{0} \\
\varepsilon_{22}^{0} \\
\varepsilon_{12}^{0}
\end{array}\right\}+z\left\{\kappa_{22}\right\}+\Psi(z)\left\{\begin{array}{l}
\kappa_{11} \\
\eta_{22} \\
\kappa_{12}
\end{array}\right\}, \\
\varepsilon_{33}=0,\left\{\begin{array}{l}
\eta_{11} \\
\varepsilon_{23} \\
\varepsilon_{13}
\end{array}\right\}=\Psi(z)_{, 3}\left\{\begin{array}{c}
\varepsilon_{23}^{0} \\
\varepsilon_{13}^{0}
\end{array}\right\},
\end{gathered}
$$

where

$$
\begin{aligned}
& \varepsilon_{11}^{0}=u_{, 1}+\frac{1}{2} w_{, 1}^{2}, \varepsilon_{22}^{0}=v_{, 2}+\frac{1}{2} w_{, 2}^{2}, \varepsilon_{23}^{0}=\varphi_{2}, \varepsilon_{13}^{0}=\varphi_{1}, \\
& \varepsilon_{12}^{0}=v_{, 1}+u_{, 2}+w_{, 1} w_{, 2}, \\
& \kappa_{11}=-w_{, 11}, \kappa_{22}=-w_{, 22}, \kappa_{12}=-2 w_{, 12}, \\
& \eta_{11}=\varphi_{1,1}, \eta_{22}=\varphi_{2,2}, \quad \eta_{12}=\varphi_{2,1}+\varphi_{1,2} .
\end{aligned}
$$

The stress-strain relations for the FGM plate are given by

$$
\begin{aligned}
& \left\{\begin{array}{c}
\sigma_{11} \\
\sigma_{22}
\end{array}\right\}=\frac{E(z)}{1-v^{2}}\left[\begin{array}{cc}
1 & v \\
v & 1
\end{array}\right]\left\{\begin{array}{c}
\varepsilon_{11}-\alpha T \\
\varepsilon_{22}-\alpha T
\end{array}\right\}, \\
& \left\{\sigma_{23}, \sigma_{13}, \sigma_{12}\right\}=\frac{E(z)}{2(1+v)}\left\{\varepsilon_{23}, \varepsilon_{13}, \varepsilon_{12}\right\},
\end{aligned}
$$

where $T(x, y, z)$ is the temperature rise through the thickness.

The stress and moment resultants of the FGM plate can be obtained by integrating Eq.9 over the thickness, and are written as

$$
\begin{aligned}
& \left\{\begin{array}{l}
N_{i} \\
M_{i} \\
S_{i}
\end{array}\right\}=\frac{1}{1-v^{2}}\left[\begin{array}{ccc}
A_{k} & B_{k} & C_{k} \\
B_{k} & D_{k} & F_{k} \\
C_{k} & F_{k} & G_{k}
\end{array}\right]\left\{\begin{array}{l}
\bar{\varepsilon}_{i}^{0} \\
\bar{\kappa}_{i} \\
\bar{\eta}_{i}
\end{array}\right\}+\frac{1}{v-1}\left[\begin{array}{c}
A_{T} \\
B_{T} \\
C_{T}
\end{array}\right], \\
& \left\{\begin{array}{l}
N_{12} \\
M_{12} \\
S_{12}
\end{array}\right\}=\frac{1}{2(1+v)}\left[\begin{array}{ccc}
A_{k} & B_{k} & C_{k} \\
B_{k} & D_{k} & F_{k} \\
C_{k} & F_{k} & G_{k}
\end{array}\right]\left\{\begin{array}{c}
\varepsilon_{12}^{0} \\
\kappa_{12} \\
\eta_{12}
\end{array}\right\},
\end{aligned}
$$




$$
\left\{\begin{array}{c}
Q_{13} \\
Q_{23}
\end{array}\right\}=\frac{H_{k}}{2(1+v)}\left\{\begin{array}{c}
\varepsilon_{13}^{0} \\
\varepsilon_{23}^{0}
\end{array}\right\},
$$

where $i=1,2$ and

$$
\begin{array}{ll}
\bar{\varepsilon}_{1}^{0}=\varepsilon_{11}^{0}+v \varepsilon_{22}^{0}, & \bar{\varepsilon}_{2}^{0}=\varepsilon_{22}^{0}+v \varepsilon_{11}^{0}, \\
\bar{\kappa}_{1}=\kappa_{11}+v \kappa_{22}, & \bar{\kappa}_{2}=\kappa_{22}+v \kappa_{11}, \\
\bar{\eta}_{1}=\eta_{11}+v \eta_{22}, & \bar{\eta}_{2}=\eta_{22}+v \eta_{11} .
\end{array}
$$

In Eqs.10-12, $N_{1}, N_{2}$, and $N_{12}$ and $M_{1}, M_{2}$, and $M_{12}$ are the basic components of stress resultants and stress couples; $S_{1}, S_{2}$, and $S_{12}$ are additional stress couples associated with the transversal shear effects; and $Q_{13}$ and $Q_{23}$ are transversal shear stress resultants. The coefficients $A_{k}, B_{k}, C_{k}, \ldots$ etc. are defined by

$$
\begin{aligned}
& \left\{A_{k}, B_{k}, D_{k}\right\}=\int_{-h / 2}^{+h / 2} E(z)\left\{1, z, z^{2}\right\} \mathrm{d} z, \\
& \left\{C_{k}, F_{k}, G_{k}\right\}=\int_{-h / 2}^{+h / 2} \Psi(z) E(z)\{1, z, \Psi(z)\} \mathrm{d} z, \\
& \left\{A_{T}, B_{T}, C_{T}\right\}=\int_{-h / 2}^{+h / 2} \alpha(z) E(z) T(x, y, z)\{1, z, \Psi(z)\} \mathrm{d} z, \\
& H_{k}=\int_{-h / 2}^{+h / 2} E(z)\left(\Psi(z)_{, 3}\right)^{2} \mathrm{~d} z .
\end{aligned}
$$

\section{EQUILIBRIUM AND STABILITY EQUATIONS}

The total potential energy of a plate subjected to thermal loads is defined as [27]

$$
V=U_{m}+U_{b}+U_{c}+U_{T},
$$

where $U_{m}, U_{b}, U_{c}$ and $U_{T}$ are membrane strain energy, bending strain energy, coupled strain energy and thermal strain energy. The strain energy for FGM plate based on the SPT is defined as given below in Eq.16.

The equilibrium and stability equations of FGM plates may be derived by the variational approach. The expansion of $V$ about the equilibrium state by the Taylor series is

$$
\Delta V=\delta V+\frac{1}{2 !} \delta^{2} V+\frac{1}{3 !} \delta^{3} V+\ldots .
$$

The governing equations of equilibrium can be derived by using the first variation $\delta V$. The non-linear equilibrium equations associated with the present SPT are

$$
N_{1,1}+N_{12,2}=0
$$

$$
\begin{gathered}
N_{12,1}+N_{2,2}=0 \\
M_{1,11}+2 M_{12,12}+M_{2,22}+N_{1} w_{, 11}+N_{2} w_{, 22}+2 N_{12} w_{, 12}=0, \\
S_{1,1}+S_{12,2}-Q_{13}=0 \\
S_{12,1}+S_{2,2}-Q_{23}=0 .
\end{gathered}
$$

To establish the stability equations, the condition $\delta^{2} V=0$ used to derive the stability equations of many practical plate buckling problems is also used here. The external load acting on the original configuration is considered to be the critical buckling temperature if the above equation $\left(\delta^{2} V=0\right)$ is satisfied. Assuming that the state of stable equilibrium of a general plate under thermal load may be designated by $u^{0}, v^{0}, w^{0}, \phi_{1}^{0}, \phi_{2}^{0}$. The displacements of the neighboring state are

$$
\begin{aligned}
& u=u^{0}+u^{1}, \quad \varphi_{1}=\varphi_{1}^{0}+\varphi_{1}^{1}, \\
& v=v^{0}+v^{1}, \quad \varphi_{2}=\varphi_{2}^{0}+\varphi_{2}^{1}, \\
& w=w^{0}+w^{1},
\end{aligned}
$$

where $u^{1}, v^{1}, w^{1}, \varphi_{1}^{1}$ and $\varphi_{2}^{1}$ are arbitrarily small increment of displacements. The stability equations are represented by using the above total displacement given in Eq.19 in the equation $\delta^{2} V=0$ and collecting the second-order terms. They read

$$
\begin{gathered}
N_{1,1}^{1}+N_{12,2}^{1}=0, \\
N_{12,1}^{1}+N_{2,2}^{1}=0, \\
M_{1,11}^{1}+2 M_{12,12}^{1}+M_{2,22}^{1}+N_{1}^{0} w_{, 11}^{1}+N_{2}^{0} w_{, 22}^{1}+2 N_{12}^{0} w_{, 12}^{1}=0, \\
S_{1,1}^{1}+S_{12,2}^{1}-Q_{13}^{1}=0, \\
S_{12,1}^{1}+S_{2,2}^{1}-Q_{23}^{1}=0,
\end{gathered}
$$

where the superscript 1 refers to the state of stability and the superscript 0 refers to the state of equilibrium conditions. The terms $N_{1}^{0}, N_{2}^{0}$ and $N_{12}^{0}$ are the pre-buckling force resultants obtained as

$$
N_{1}^{0}=\frac{A_{T}}{v-1}, N_{1}^{0}=\frac{A_{T}}{v-1}, N_{12}^{0}=0 .
$$

\section{EXACT SOLUTIONS FOR THERMAL BUCKLING OF FGM PLATES}

Rectangular plates are generally classified in accordance

$$
V=\frac{1}{2} \iiint_{\mathrm{v}}\left[\sigma_{11}\left(\varepsilon_{11}-\alpha T\right)+\sigma_{22}\left(\varepsilon_{22}-\alpha T\right)+\sigma_{12} \varepsilon_{12}+\sigma_{23} \varepsilon_{23}+\sigma_{13} \varepsilon_{13}\right] \mathrm{dv}
$$


with the type support used in the absent of the body forces and lateral loads except the external temperature load. The following boundary conditions are imposed at the side edges

$$
\begin{aligned}
& v^{1}=w^{1}=\varphi_{2}^{1}=N_{1}^{1}=M_{1}^{1}=S_{1}^{1}=0 \text { at } x=0, a, \\
& u^{1}=w^{1}=\varphi_{1}^{1}=N_{2}^{1}=M_{2}^{1}=S_{2}^{1}=0 \text { at } y=0, b,
\end{aligned}
$$

Following Navier solution procedure, we assume the following solution form for $\left(u^{1}, v^{1}, w^{1}, \varphi_{1}^{1}, \varphi_{2}^{1}\right)$ that satisfies the simply-supported boundary conditions,

$$
\left\{\begin{array}{l}
u^{1} \\
v^{1} \\
w^{1} \\
\varphi_{1}^{1} \\
\varphi_{2}^{1}
\end{array}\right\}=\sum_{m, n=1}^{\infty}\left\{\begin{array}{c}
U_{m n}^{1} \cos (\lambda x) \sin (\mu y) \\
V_{m n}^{1} \sin (\lambda x) \cos (\mu y) \\
W_{m n}^{1} \sin (\lambda x) \sin (\mu y) \\
X_{m n}^{1} \cos (\lambda x) \sin (\mu y) \\
Y_{m n}^{1} \sin (\lambda x) \cos (\mu y)
\end{array}\right\},
$$

where $\lambda=m \pi / a, \mu=n \pi / b ; m$ and $n$ are mode numbers; $U_{m n}^{1}, V_{m n}^{1}, W_{m n}^{1}, X_{m n}^{1}$, and $Y_{m n}^{1}$ are arbitrary parameters to be determined subjected to the condition that the solution in Eq.23 satisfies the conditions in Eq.22. Substituting Eq.23 into Eq.20, one obtains

$$
[L]\{\Omega\}=0,
$$

where $\{\Omega\}$ denotes the column

$$
\{\Omega\}^{t}=\left\{U_{m n}^{1}, V_{m n}^{1}, W_{m n}^{1}, X_{m n}^{1}, Y_{m n}^{1}\right\},
$$

and elements $L_{r s}=L_{s r}$ of the coefficient matrix [L] are given by:

$$
\begin{gathered}
L_{11}=-A_{k}\left[2 \lambda^{2}+(1-v) \mu^{2}\right], \\
L_{12}=-\lambda \mu A_{k}(1+v), \\
L_{13}=2 \lambda B_{k}\left(\lambda^{2}+\mu^{2}\right), \\
L_{14}=-C_{k}\left[2 \lambda^{2}+(1-v) \mu^{2}\right], \\
L_{15}=-\lambda \mu C_{k}(1+v), \\
L_{22}=-A_{k}\left[(1-v) \lambda^{2}+2 \mu^{2}\right], \\
L_{23}=2 \mu B_{k}\left(\lambda^{2}+\mu^{2}\right), \\
L_{24}=L_{15}, \\
L_{25}=-C_{k}\left[(1-v) \lambda^{2}+2 \mu^{2}\right], \\
L_{33}=-2\left(\lambda^{2}+\mu^{2}\right)\left[D_{k}\left(\lambda^{2}+\mu^{2}\right)-A_{T}(1+v)\right], \\
L_{34}=2 \lambda F_{k}\left(\lambda^{2}+\mu^{2}\right),
\end{gathered}
$$

$$
\begin{gathered}
L_{35}=2 \mu F_{k}\left(\lambda^{2}+\mu^{2}\right), \\
L_{44}=-G_{k}\left[2 \lambda^{2}+(1-v) \mu^{2}\right]-H_{k}(1-v), \\
L_{45}=-\lambda \mu G_{k}(1+v), \\
L_{55}=-G_{k}\left[(1-v) \lambda^{2}+2 \mu^{2}\right]-H_{k}(1-v) .
\end{gathered}
$$

For non-trivial solutions of Eq.24, the determinant $|L|$ should be zero. This equation $(|L|=0)$ is stated for the determination of the lowest critical load. In the following, the solutions of the equation $|L|=0$ for different types of thermal loading conditions are presented. The plate is assumed simply supported in bending and rigidly fixed in extension. The temperature change is varied only in the thickness direction.

\subsection{Thermal Buckling for FGM Plates under Uniform Temperature Rise}

The initial uniform temperature of the plate is assumed to be $T_{i}$. The temperature is uniformly raised to a final value $T_{f}$ in which the plate buckles. The temperature change is $\Delta T=T_{f}-T_{i}$. Substituting Eq.26 into the equation $|L|=0$, the buckling temperature change using the shear deformation theories is obtained as

$$
\Delta T=\frac{\pi^{2}\left(n^{2} s^{2}+m^{2}\right)\left[\bar{P}_{1} a^{2}(1-v)+\bar{P}_{2} \pi^{2}\left(n^{2} s^{2}+m^{2}\right)\right]}{a^{2} A_{T 1}(1+v)\left[P_{1} a^{2}(1-v)+P_{2} \pi^{2}\left(n^{2} s^{2}+m^{2}\right)\right]},
$$

where

$$
\begin{gathered}
P_{1}=A_{k} H_{k}, P_{2}=2\left(A_{k} G_{k}-C_{k}^{2}\right), \\
\bar{P}_{1}=P_{1} D_{k}-B_{k}^{2} H_{k}, \\
\bar{P}_{2}=P_{2} D_{k}-2 A_{k} F_{k}^{2}-2 B_{k}\left(B_{k} G_{k}-2 F_{k} C_{k}\right), \\
A_{T 1}=\int_{-h / 2}^{+h / 2} \alpha(z) E(z) \mathrm{d} z, s=a / b .
\end{gathered}
$$

The critical buckling temperature change $T_{c r}$, is the smallest value of $\Delta T$ which is obtained when $m=1$ and $n=1$. Therefore,

$$
T_{c r}=\frac{\pi^{2}\left(s^{2}+1\right)\left[\bar{P}_{1} a^{2}(1-v)+\bar{P}_{2} \pi^{2}\left(s^{2}+1\right)\right]}{a^{2} A_{T 1}(1+v)\left[P_{1} a^{2}(1-v)+P_{2} \pi^{2}\left(s^{2}+1\right)\right]} .
$$

For the classical plate theory, the critical buckling temperature difference $T_{c r}$ is given as

$$
T_{c r}=\frac{\pi^{2}\left(s^{2}+1\right)\left(A_{k} D_{k}-B_{k}\right)}{a^{2} A_{k} A_{T 1}(1+v)} .
$$

\subsection{Thermal Buckling for FGM Plates Subjected to a Graded Temperature Change across the Thickness}

For an FG plate, the temperature change is not uniform. The temperature varies according to the power law variation. Usually, the temperature rises much higher at 
the ceramic side than that in the metal side of the plate. In this case, the temperature through the thickness is given by

$$
T(z)=\Delta T V^{\beta}+T_{\mathrm{m}},
$$

where $T_{\mathrm{m}}$ is the temperature of the bottom surface which is metal-rich and $\beta$ is the power law exponent $(0<\beta<\infty)$.

Similar to the previous loading case, solving the equation $|L|=0$, the buckling temperature difference $\Delta T=T(h / 2)-T(-h / 2)$ using the shear deformation plate theories can be determined, and then we can obtain the critical buckling $T_{c r}$ as

$$
T_{c r}=\frac{\pi^{2}\left(s^{2}+1\right)\left[\bar{P}_{1} a^{2}(1-v)+\bar{P}_{2} \pi^{2}\left(s^{2}+1\right)\right]}{a^{2} A_{T 2}(1+v)\left[P_{1} a^{2}(1-v)+P_{2} \pi^{2}\left(s^{2}+1\right)\right]}-\frac{T_{m} A_{T 1}}{A_{T 2}},
$$

where

$$
A_{T 2}=\int_{-h / 2}^{+h / 2} \alpha(z) V^{\beta} E(z) \mathrm{d} z .
$$

Also, the critical buckling temperature difference $T_{c r}$ for the classical plate theory, is deduced as

$$
T_{c r}=\frac{\pi^{2}\left(s^{2}+1\right)\left(A_{k} D_{k}-B_{k}\right)}{a^{2} A_{k} A_{T 2}(1+v)}-\frac{T_{m} A_{T 1}}{A_{T 2}} .
$$

Note that the value of $\beta$ equal to unity represents a linear temperature change across the thickness. While the value of $\beta$ excluding unity represents a non-linear temperature change through the thickness.

\section{RESULTS AND DISCUSSION}

The general approach outlined in the previous sections for the thermal buckling analysis of the homogeneous and FGM plates under uniform, linear and non-linear temperature rises through the thickness is illustrated in this section using the SPT. The correlation between the present theory and different higher- and first-order shear deformation theories and classical plate theory is established. To illustrate the proposed method, a ceramic-metal FG plate is considered. The combination of materials consists of aluminum and alumina. The Young's modulus and the coefficient of thermal expansion for alumina are $E_{\mathrm{c}}=380 \mathrm{GPa}, \alpha_{\mathrm{c}}=7.4 \times 10^{-6} /{ }^{\circ} \mathrm{C}$, and for aluminum are $E_{\mathrm{m}}=70 \mathrm{GPa}, \alpha_{\mathrm{m}}=23 \times 10^{-6} /{ }^{\circ} \mathrm{C}$, respectively. Note that, Poisson's ratio is selected constant for both aluminum and alumina and it equal to 0.3 . The shear correction factor for FPT is set equal to 5/6. For the linear and non-linear temperature rises through the thickness, the temperature rises $5^{\circ} \mathrm{C}$ in the metal-rich surface of the plate (i.e. $T_{\mathrm{m}}=5^{\circ} \mathrm{C}$ ). We will assume in all analyzed cases (unless otherwise stated) that $a / b=2$, $a / h=10$, and $\beta=3$.

Numerical results of the present investigation are given in Tables 1-4 and Figures 1-4. In Tables 1 and 2, the side to thickness ratio of the plate is set as $a / h=100$. In these tables the critical buckling temperature difference $T_{c r}$ of the plate under uniform and linear temperature rises is shown for different values of the power law index $k$ using various plate theories. The results obtained as per the present HPT and CPT are compared with the corresponding ones presented by Javaheri and Eslami [32]. Excellent agreement is achieved between the two solutions. It is seen that, for all theories, the critical temperature difference increases monotonically as the aspect ratio $a / b$ increases. Moreover, the critical buckling $T_{c r}$ decreases until it reaches minimum values and then increases as the values of the volume fraction exponent $k$ increases. Tables 3 and $\mathbf{4}$ exhibit the critical temperature difference $t_{c r}=10^{-3} T_{c r}$ for different values of the aspect ratio $a / b$, the temperature exponent $\beta$ and the power law index $k$ under non-linear temperature loads at $a / h=10$ and 5 , respectively. The nonlinearity temperature exponent $\beta$ is taken here as 2 , 5 and 10 . The effect of $a / b$ on the critical buckling $t_{c r}$ is similar to that in the case of uniform and linear temperature difference across the thickness. As the power law index $k$ increases, the critical buckling $t_{c r}$ decreases to reach lowest values and then increases excluding $t_{c r}$ of the rectangular plates for $\beta=10$. Also, it is noticed that $t_{c r}$ increases as the nonlinearity index $\beta$ increases. In general, the values of the critical temperature difference calculated by using the shear deformation theories are lower than those calculated by using the classical plate theory, indicating the shear deformation effect. The SPT without using any shear correction factor gives results very close to HPT and closer than those obtained using FPT.

The critical buckling temperature difference $t_{c r}$ of the ceramic-metal FG rectangular plate $(k=5)$ versus the side-to-thickness ratio $a / h$ calculated by all theories under a uniform, linear and non-linear temperature load are shown in Figure 1. For plates with small $a / h$ ratio, very large differences between the results of both SPT and HPT and those of both FPT and CPT are observed. Moreover, the differences between the higherorder shear deformation theories (SPT and HPT) and FPT are lower than those between any of them and CPT. However, for a large value of the side-to-thickness ratio the difference between the values predicted by the shear deformation theories and CPT is low significant because the plate is essentially thin. Because of permitting shear deformation in SPT, HPT and FPT, the plate becomes more flexible and thus the critical buckling temperatures calculated by these theories are smaller than those cal- 
Table 1. Critical buckling temperature change $T_{c r}$ of FGM plate under uniform temperature rise for different values of power law index $k$ and aspect ratio $a / b$.

\begin{tabular}{ccccccc}
\hline \multirow{2}{*}{$k$} & Theory & $a / b=1$ & $a / b=2$ & $a / b=3$ & $a / b=4$ & $a / b=5$ \\
\hline \multirow{4}{*}{0} & SPT & 17.0894 & 42.6876 & 85.2554 & 144.6500 & 220.6729 \\
& HPT & $17.0894(17.088)$ & $42.6875(42.688)$ & $85.2551(85.252)$ & $144.6490(144.648)$ & $220.6706(220.667)$ \\
& FPT & 17.0894 & 42.6875 & 85.2551 & 144.6489 & 220.6704 \\
& CPT & $17.0991(17.099)$ & $42.7477(42.747)$ & $85.4955(85.495)$ & $145.3424(145.342)$ & $222.2883(222.288)$ \\
& SPT & 7.9400 & 19.8359 & 39.6249 & 67.2510 & 102.6365 \\
1 & HPT & $7.9400(7.939)$ & $19.8358(19.835)$ & $39.6248(39.624)$ & $67.2506(67.250)$ & $102.6356(102.634)$ \\
& FPT & 7.9400 & 19.8358 & 39.6248 & 67.2506 & 102.6355 \\
& CPT & $7.9437(7.943)$ & $19.8594(19.859)$ & $39.7188(39.718)$ & $67.5220(67.522)$ & $103.2690(103.269)$ \\
& SPT & 7.0390 & 17.5840 & 35.1233 & 59.6034 & 90.9501 \\
2 & HPT & 7.0390 & 17.5840 & 35.1234 & 59.6037 & 90.9508 \\
& FPT & 7.0392 & 17.5853 & 35.1285 & 59.6184 & 91.9850 \\
& CPT & 7.0426 & 17.6065 & 35.2130 & 59.8621 & 91.5538 \\
& SPT & 7.2606 & 18.1324 & 36.2014 & 61.3921 & 93.5999 \\
5 & HPT & $7.2606(7.260)$ & $18.1327(18.132)$ & $36.2025(36.203)$ & $61.3951(61.395)$ & $93.6069(93.605)$ \\
& FPT & 7.2615 & 18.1380 & 36.2236 & 61.4559 & 93.7481 \\
& CPT & $7.2657(7.265)$ & $18.1642(18.164)$ & $36.3285(36.328)$ & $61.7585(61.758)$ & $94.4542(94.454)$ \\
& SPT & 7.4634 & 18.6365 & 37.2001 & 63.0673 & 96.1183 \\
10 & HPT & $7.4634(7.462)$ & $18.6366(18.636)$ & $37.2006(37.200)$ & $63.0687(63.068)$ & $96.1213(96.120)$ \\
& FPT & 7.4644 & 18.6427 & 37.2246 & 63.1378 & 96.2820 \\
& CPT & $7.4692(7.469)$ & $18.6731(18.673)$ & $37.3463(37.346)$ & $63.4888(63.488)$ & $97.1005(97.100)$ \\
\hline
\end{tabular}

The results in parenthesis are obtained in [32].

Table 2. Critical buckling temperature change $T_{c r}$ of FGM plate under linear temperature rise for different values of power law index $k$ and aspect ratio $a / b$.

\begin{tabular}{ccccccc}
\hline \multirow{2}{*}{$k$} & Theory & $a / b=1$ & $a / b=2$ & $a / b=3$ & $a / b=4$ & $a / b=5$ \\
\hline & SPT & 24.1789 & 75.3753 & 160.5109 & 279.3000 & 431.3459 \\
0 & HPT & $24.1789(24.177)$ & $75.3751(75.376)$ & $160.5102(160.505)$ & $279.2980(279.297)$ & $431.3412(431.334)$ \\
& FPT & 24.1789 & 75.3751 & 160.5102 & 279.2979 & 431.3409 \\
& CPT & $24.1982(24.198)$ & $75.4955(75.495)$ & $160.9910(160.991)$ & $280.6848(280.684)$ & $434.5767(434.576)$ \\
& SPT & 5.5138 & 27.8242 & 64.9379 & 116.7498 & 183.1140 \\
& HPT & $5.5138(5.513)$ & $27.8242(27.823)$ & $64.9376(64.936)$ & $116.7490(116.748)$ & $183.1123(183.110)$ \\
1 & FPT & 5.5138 & 27.8242 & 64.9376 & 116.7490 & 183.1122 \\
& CPT & $5.5209(5.520)$ & $27.8683(27.868)$ & $65.1140(65.114)$ & $117.2580(117.258)$ & $184.3002(184.300)$ \\
& SPT & 3.5893 & 22.1521 & 53.0271 & 96.1203 & 151.3011 \\
2 & HPT & 3.5893 & 22.1522 & 53.0273 & 96.1209 & 151.3023 \\
& FPT & 3.5897 & 22.1544 & 53.0363 & 96.1467 & 151.3624 \\
& CPT & 3.5956 & 22.1916 & 53.1850 & 96.5757 & 152.3637 \\
& SPT & 3.8911 & 22.6047 & 53.7068 & 97.0673 & 152.5063 \\
5 & HPT & $3.8912(3.891)$ & $22.6052(22.604)$ & $53.7086(53.710)$ & $97.0725(97.073)$ & $152.5184(152.516)$ \\
& FPT & 3.8927 & 22.6143 & 53.7450 & 97.1771 & 152.7615 \\
& CPT & $3.8999(3.899)$ & $22.6595(22.659)$ & $53.9256(53.925)$ & $97.6980(97.698)$ & $153.9769(153.977)$ \\
& SPT & 4.3653 & 24.1648 & 57.0607 & 102.8991 & 161.4674 \\
10 & HPT & $4.3653(4.364)$ & $24.1650(24.165)$ & $57.0615(57.061)$ & $102.9015(102.901)$ & $161.4729(161.471)$ \\
& FPT & 4.3670 & 24.1757 & 57.1041 & 103.0240 & 161.7575 \\
& CPT & $4.3757(4.375)$ & $24.2297(24.229)$ & $57.3198(57.319)$ & $103.6459(103.646)$ & $163.2080(163.208)$ \\
\hline
\end{tabular}

The results in parenthesis are obtained in [32].

culated by CPT.

The critical buckling temperature difference $t_{c r}$ as a function of $a / b$ for various values of the power law index $k$ under a uniform, linear and non-linear temperature loads is depicted in Figure 2. It is observed that, with increasing the plate aspect ratio $a / b$, the critical buckling temperature difference also increases gradually, whatever the material gradient index $k$ is. Since the ceramic plate is weaker than the metallic one, thus the critical buckling temperature of the first plate is higher 
Table 3. Critical buckling temperature change $t_{c r}$ of FGM plate under non-linear temperature rise for different values of index $k$, aspect ratio $a / b$, and temperature exponent $\beta \quad(a / h=10)$.

\begin{tabular}{|c|c|c|c|c|c|c|c|c|c|c|}
\hline \multirow{2}{*}{$k$} & \multirow{2}{*}{ Theory } & \multicolumn{3}{|c|}{$a / b=1$} & \multicolumn{3}{|c|}{$a / b=2$} & \multicolumn{3}{|c|}{$a / b=3$} \\
\hline & & $\beta=2$ & $\beta=5$ & $\beta=10$ & $\beta=2$ & $\beta=5$ & $\beta=10$ & $\beta=2$ & $\beta=5$ & $\beta=10$ \\
\hline \multirow{4}{*}{0} & SPT & 4.8414 & 9.6829 & 17.7520 & 11.2294 & 22.4589 & 41.1747 & 20.0164 & 40.0328 & 73.3935 \\
\hline & HPT & 4.8410 & 9.6821 & 17.7505 & 11.2269 & 22.4538 & 41.1654 & 20.0066 & 40.0133 & 73.3577 \\
\hline & FPT & 4.8408 & 9.6817 & 17.7498 & 11.2246 & 22.4492 & 41.1568 & 19.9919 & 39.9838 & 73.3037 \\
\hline & CPT & 5.1147 & 10.2294 & 18.7540 & 12.8093 & 25.6186 & 46.9675 & 25.6336 & 51.2673 & 93.9900 \\
\hline \multirow{4}{*}{1} & SPT & 2.1068 & 4.3182 & 8.1906 & 4.9517 & 10.1496 & 19.2512 & 8.9711 & 18.3880 & 34.8774 \\
\hline & HPT & 2.1066 & 4.3179 & 8.1900 & 4.9508 & 10.1476 & 19.2475 & 8.9673 & 18.3802 & 34.8626 \\
\hline & FPT & 2.1065 & 4.3178 & 8.1898 & 4.9499 & 10.1458 & 19.2440 & 8.9615 & 18.3684 & 34.8402 \\
\hline & $\mathrm{CPT}$ & 2.2072 & 4.5241 & 8.5812 & 5.5391 & 11.3534 & 21.5346 & 11.0921 & 22.7355 & 43.1235 \\
\hline \multirow{4}{*}{2} & SPT & 1.6765 & 3.2736 & 6.1232 & 3.9243 & 7.6627 & 14.3327 & 7.0655 & 13.7962 & 25.8051 \\
\hline & HPT & 1.6766 & 3.2738 & 6.1235 & 3.9246 & 7.6633 & 14.3339 & 7.0659 & 13.7970 & 25.8066 \\
\hline & FPT & 1.6812 & 3.2828 & 6.1404 & 3.9493 & 7.7116 & 14.4242 & 7.1433 & 13.9483 & 26.0895 \\
\hline & $\mathrm{CPT}$ & 1.7627 & 3.4419 & 6.4379 & 4.4256 & 8.6417 & 16.1638 & 8.8640 & 17.3080 & 32.3737 \\
\hline \multirow{4}{*}{5} & SPT & 1.5955 & 2.8485 & 4.9990 & 3.6479 & 6.5126 & 11.4292 & 6.3635 & 11.3609 & 19.9377 \\
\hline & HPT & 1.5964 & 2.8500 & 5.0017 & 3.6521 & 6.5202 & 11.4425 & 6.3755 & 11.3822 & 19.9751 \\
\hline & FPT & 1.6141 & 2.8816 & 5.0571 & 3.7444 & 6.6849 & 11.7317 & 6.6569 & 11.8847 & 20.8569 \\
\hline & $\mathrm{CPT}$ & 1.7083 & 3.0498 & 5.3522 & 4.2885 & 7.6562 & 13.4363 & 8.5888 & 15.3337 & 26.9097 \\
\hline \multirow{4}{*}{10} & SPT & 1.6766 & 2.8844 & 4.7717 & 3.7953 & 6.5293 & 10.8015 & 6.5362 & 11.2448 & 18.6022 \\
\hline & HPT & 1.6770 & 2.8851 & 4.7728 & 3.7970 & 6.5322 & 10.8062 & 6.5402 & 11.2515 & 18.6134 \\
\hline & FPT & 1.6974 & 2.9202 & 4.8310 & 3.9016 & 6.7122 & 11.1040 & 6.8510 & 11.7862 & 19.4980 \\
\hline & CPT & 1.8092 & 3.1126 & 5.1492 & 4.5414 & 7.8130 & 12.9250 & 9.0951 & 15.6470 & 25.8848 \\
\hline
\end{tabular}

Table 4. Critical buckling temperature change $t_{c r}$ of FGM plate under non-linear temperature rise for different values of index $k$, aspect ratio $a / b$, and temperature exponent $\beta \quad(a / h=5)$.

\begin{tabular}{|c|c|c|c|c|c|c|c|c|c|c|}
\hline \multirow{2}{*}{$k$} & \multirow{2}{*}{ Theory } & \multicolumn{3}{|c|}{$a / b=1$} & \multicolumn{3}{|c|}{$a / b=2$} & \multicolumn{3}{|c|}{$a / b=3$} \\
\hline & & $\beta=2$ & $\beta=5$ & $\beta=10$ & $\beta=2$ & $\beta=5$ & $\beta=10$ & $\beta=2$ & $\beta=5$ & $\beta=10$ \\
\hline \multirow{4}{*}{0} & SPT & 16.7416 & 33.4833 & 61.3861 & 32.8985 & 65.7971 & 120.6281 & 48.6540 & 97.3080 & 178.3980 \\
\hline & HPT & 16.7353 & 33.4706 & 61.3628 & 32.8633 & 65.7266 & 120.4989 & 48.5388 & 97.0776 & 177.9756 \\
\hline & FPT & 16.7270 & 33.4541 & 61.3325 & 32.7842 & 65.5685 & 120.2090 & 48.1978 & 96.3955 & 176.7252 \\
\hline & $\mathrm{CPT}$ & 20.5039 & 41.0078 & 75.1810 & 51.2823 & 102.5646 & 188.0351 & 102.5796 & 205.1592 & 376.1253 \\
\hline \multirow{4}{*}{1} & SPT & 7.4586 & 15.2878 & 28.9971 & 15.0945 & 30.9390 & 58.6835 & 22.9714 & 47.0843 & 89.3070 \\
\hline & HPT & 7.4561 & 15.2827 & 28.9875 & 15.0800 & 30.9094 & 58.6274 & 22.9214 & 46.9819 & 89.1127 \\
\hline & FPT & 7.4529 & 15.2762 & 28.9751 & 15.0476 & 30.8430 & 58.5014 & 22.7734 & 46.6785 & 88.5373 \\
\hline & $\mathrm{CPT}$ & 8.8709 & 18.1827 & 34.4879 & 22.1983 & 45.4997 & 86.3014 & 44.4106 & 91.0281 & 172.6573 \\
\hline \multirow{4}{*}{2} & SPT & 5.8880 & 11.4979 & 21.5048 & 11.7774 & 22.9970 & 43.0146 & 17.7227 & 34.6058 & 64.7282 \\
\hline & HPT & 5.8885 & 11.4981 & 21.5065 & 11.7751 & 22.9923 & 43.0059 & 17.7018 & 34.5650 & 64.6519 \\
\hline & FPT & 5.9430 & 11.6045 & 21.7057 & 11.9755 & 23.3838 & 43.7381 & 18.0864 & 35.3160 & 66.0566 \\
\hline & $\mathrm{CPT}$ & 7.0886 & 13.8415 & 25.8898 & 17.7406 & 34.6407 & 64.7936 & 35.4938 & 69.3061 & 129.6333 \\
\hline \multirow{4}{*}{5} & SPT & 5.3654 & 9.5789 & 16.8104 & 10.1426 & 18.1076 & 31.7779 & 14.4932 & 25.8748 & 45.4087 \\
\hline & HPT & 5.3742 & 9.5945 & 16.8378 & 10.1682 & 18.1534 & 31.8582 & 14.5269 & 25.9349 & 45.5142 \\
\hline & FPT & 5.5741 & 9.9515 & 17.4644 & 10.8794 & 19.4230 & 34.0863 & 15.9245 & 28.4301 & 49.8932 \\
\hline & $\mathrm{CPT}$ & 6.8687 & 12.2627 & 21.5203 & 17.1895 & 30.6885 & 53.8566 & 34.3909 & 61.3982 & 107.7502 \\
\hline \multirow{4}{*}{10} & SPT & 5.5369 & 9.5255 & 15.7580 & 10.2387 & 17.6144 & 29.1395 & 14.3554 & 24.6965 & 40.8554 \\
\hline & HPT & 5.5400 & 9.5308 & 15.7669 & 10.2435 & 17.6226 & 29.1530 & 14.3463 & 24.6810 & 40.8297 \\
\hline & FPT & 5.7630 & 9.9144 & 16.4014 & 11.0005 & 18.9250 & 31.3076 & 15.7723 & 27.1342 & 44.8880 \\
\hline & $\mathrm{CPT}$ & 7.2736 & 12.5134 & 20.7009 & 18.2025 & 31.3150 & 51.8043 & 36.4172 & 62.6510 & 103.6433 \\
\hline
\end{tabular}

than that of the second. For the FGM plate, $t_{c r}$ decreases as the metallic constituent in the plate increases.

Figure 3 investigates the critical buckling temperature difference $t_{c r}$ of homogeneous and FG plates versus the side-to-thickness ratio $a / h$ under various types of temperature loads. Figure 4 gives similar for FG plates versus the aspect ratio $a / b$. The buckling temperature of the homogeneous plate is considerably higher than 

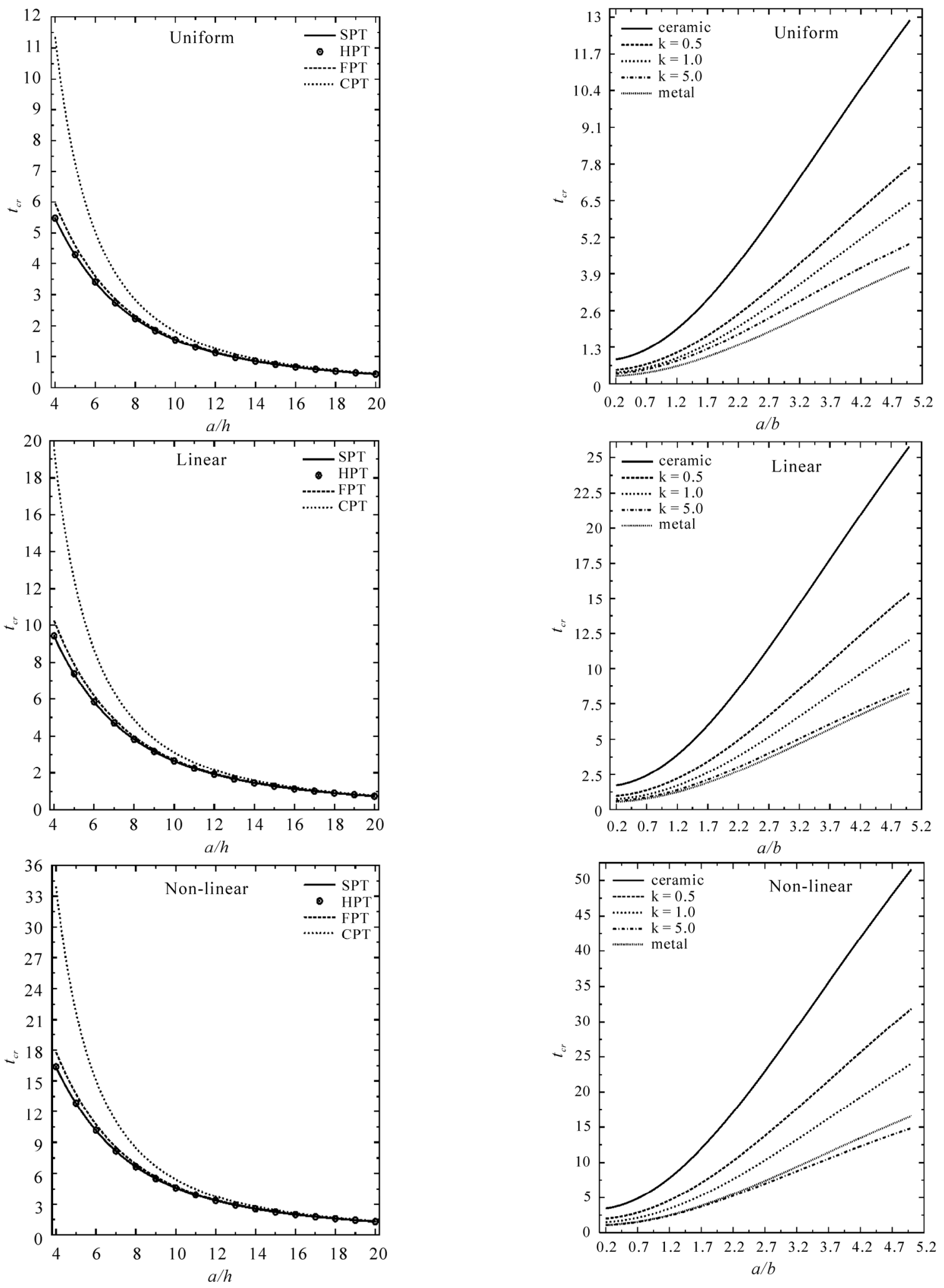

Figure 1. Critical buckling temperature difference $t_{c r}$ due to uniform, linear and non-linear temperature rise across the thickness versus the side-tothickness ratio $a / h$.

Figure 2. Critical buckling temperature difference $t_{c r}$ due to uniform, linear and non-linear temperature rise across the thickness versus the aspect ratio $a / b$. 


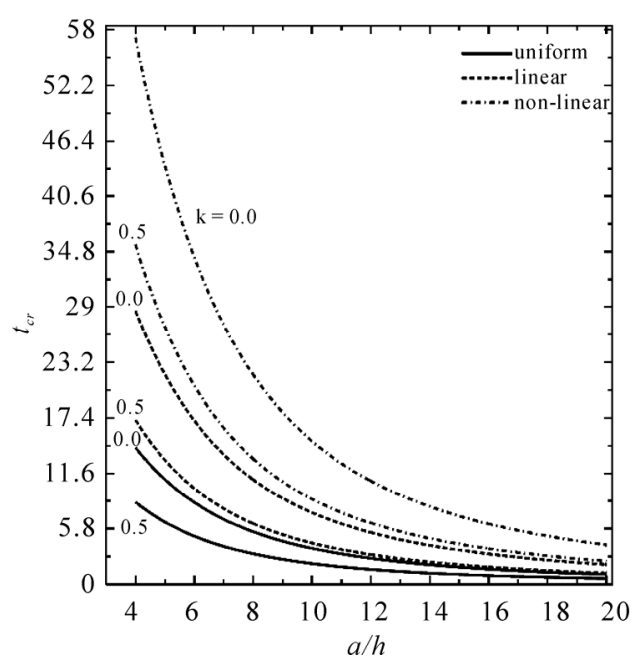

Figure 3. Critical buckling temperature difference $t_{c r}$ due to uniform, linear and non-linear temperature rise across the thickness versus the side-tothickness ratio $a / h$.

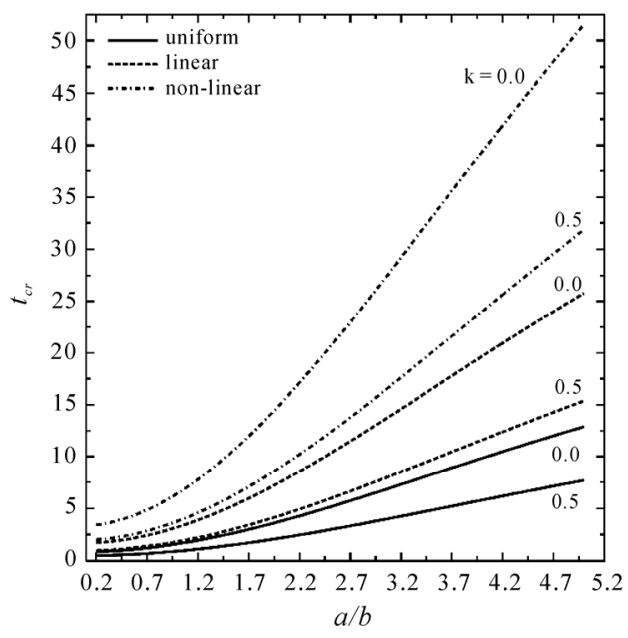

Figure 4. Critical buckling temperature difference $t_{c r}$ due to uniform, linear and non-linear temperature rise across the thickness versus the aspect ratio $a / b$.

that for the FGM one, especially for the comparatively thicker plates. Again, because of the thicker plates are stronger than the thinner ones, thus the critical buckling temperature of the first type is higher than that of the second one. Note that $t_{c r}$ of the plate under uniform temperature rise is smaller than that of the plate under linear temperature rise and the latter is smaller than that of the plate under non-linear temperature rise.

\section{CONCLUSIONS}

The thermal buckling analysis for ceramic-metal FG plates under uniform, linear and nonlinear thermal loading through the thickness is investigated in this paper. The constituent materials are graded from the ceramic surface to the metallic surface according to the power law variation. The SPT is used to deduce the equilibrium and stability equations for a simply supported functionally graded rectangular plate under thermal loading. The results obtained using SPT are compared with those obtained using HPT, FPT and CPT; and compared with published ones. The numerical results of critical buckling temperature difference using SPT are very close to those of HPT and the two theories have similar trends for all cases of loading. The critical buckling temperature difference is proportional to the plate aspect ratio. The thicker plates need a temperature to buckle higher than that the thinner plates need it. The critical buckling temperature differences of functionally graded plates are generally lower than the corresponding ones for homogeneous ceramic plates.

\section{ACKNOWLEDGEMENTS}

The investigators would like to express their appreciation to the Deanship of Scientific Research at King AbdulAziz University for its financial support of this study, Grant No. 3-038/429.

\section{REFERENCES}

[1] Koizumi, M. (1997) FGM activities in Japan. Composites Part B: Engineering, 28(1-2), 1-4.

[2] Bhangale, R.K. and Ganesan, N. (2005) A linear thermoelastic buckling behavior of functionally graded hemispherical shell with a cut-out at apex in thermal environment. International Journal of Structural Stability and Dynamics, 5(2), 185-215.

[3] Javaheri, R. and Eslami, M.R. (2002) Buckling of functionally graded plates under in-plane compressive loading. ZAMM - Journal of Applied Mathematics and Mechanics, 82(4), 277-283.

[4] Chung, Y.-L. and Chang, H.-X. (2008) Mechanical behavior of rectangular plates with functionally graded coefficient of thermal expansion subjected to thermal loading. Journal of Thermal Stresses, 31(4), 368-388.

[5] Praveen, G.N. and Reddy, J.N. (1998) Nonlinear transient thermoelastic analysis of functionally graded ceramicmetal plates. International Journal of Solids and Structures, 35(33), 4457-4476.

[6] Reddy, J.N. (2000) Analysis of functionally graded plates. International Journal for Numerical Methods in Engineering, 47(1-3), 663-684.

[7] Amini, M.H., Soleimani, M. and Rastgoo, A. (2009) Three-dimensional free vibration analysis of functionally graded material plates resting on an elastic foundation. Smart Materials and Structures, 18(8), 1-9.

[8] Sladek, J., Sladek, V., Hellmich, Ch. and Eberhardsteiner, J. (2007) Analysis of thick functionally graded plates by local integral equation method. Communications in $\mathrm{Nu}$ merical Methods in Engineering, 23(8), 733-754. 
[9] Kim, J.-H. and Paulino, G.H. (2002) Finite element evaluation of mixed mode stress intensity factors in functionally graded materials. International Journal for $\mathrm{Nu}$ merical Methods in Engineering, 53(8), 1903-1935.

[10] Altenbach, H. and Eremeyev, V.A. (2008) Analysis of the viscoelastic behavior of plates made of functionally graded materials. ZAMM - Journal of Applied Mathematics and Mechanics, 88(5), 332-341.

[11] Ovesy, H.R. and Ghannadpour, S.A.M. (2007) Large deflection finite strip analysis of functionally graded plates under pressure loads. International Journal of Structural Stability and Dynamics, 7(2), 193-211.

[12] Han, X., Liu, G.R. and Lam, K.Y. (2001) Transient waves in plates of functionally graded materials. International Journal for Numerical Methods in Engineering, 52(8), 851-865.

[13] Zenkour, A.M. (2007) Benchmark trigonometric and 3-D elasticity solutions for an exponentially graded thick rectangular plate. Archive of Applied Mechanics, 77(4), 197-214.

[14] Zenkour, A.M. (2005) A comprehensive analysis of functionally graded sandwich plates: Part 1-Deflection and stresses. International Journal of Solids and Structures, 42(18-19), 5224-5242.

[15] Zenkour, A.M. (2005) A comprehensive analysis of functionally graded sandwich plates: Part 2-Buckling and free vibration. International Journal of Solids and Structures, 42(18-19), 5243-5258.

[16] Zenkour, A.M. (2005) On vibration of functionally graded plates according to a refined trigonometric plate theory. International Journal of Structural Stability and Dynamics, 5(2), 279-297.

[17] Aliaga, J.W. and Reddy, J.N. (2004) Nonlinear thermoelastic analysis of functionally graded plates using the third-order shear deformation theory. International Journal of Computational Engineering Science, 5(4), 753779.

[18] Arciniega, R.A. and Reddy, J.N. (2007) Large deformation analysis of functionally graded shells. International Journal of Solids and Structures, 44(6), 2036-2052.

[19] Kadoli, R., Akhtar, K. and Ganesan, N. (2008) Static analysis of functionally graded beams using higher order shear deformation theory. Applied Mathematical Modelling, 32(12), 2509-2525.
[20] Sina, S.A., Navazi, H.M. and Haddadpour, H. (2009) An analytical method for free vibration analysis of functionally graded beams. Materials \& Design, 30(3), 741-747.

[21] Zhao, X., Lee, Y.Y. and Liew, K.M. (2009) Mechanical and thermal buckling analysis of functionally graded plates. Composite Structures, 90(2), 161-171.

[22] Matsunaga, H. (2009) Thermal buckling of functionally graded plates according to a 2D higher-order deformation theory. Composite Structures, 90(1), 76-86.

[23] Morimoto, T., Tanigawa, Y. and Kawamura, R. (2006) Thermal buckling of functionally graded rectangular plates subjected to partial heating. International Journal of Mechanical Sciences, 48(9), 926-937.

[24] Shariat, B.A.S. and Eslami, M.R. (2006) Thermal buckling of imperfect functionally graded plates. International Journal of Solids and Structures, 43(14-15), 40824096.

[25] Ganapathi, M. and Prakash, T. (2006) Thermal buckling of simply supported functionally graded skew plates. Composite Structures, 74(2), 247-250.

[26] Lanhe, W. (2004) Thermal buckling of a simply supported moderately thick rectangular FGM plate. Composite Structures, 64(2), 211-218.

[27] Najafizadeh, M.M. and Heydari, H.R. (2004) Thermal buckling of functionally graded circular plates based on higher order shear deformation plate theory. European Journal of Mechanics A/Solids, 23(6), 1085-1100.

[28] Babu, C.S. and Kant, T. (2000) Refined higher order finite element models for thermal buckling of laminated composite and sandwich plates. Journal of Thermal Stresses, 23(2), 111-130.

[29] Zenkour, A.M. (2006) Generalized shear deformation theory for bending analysis of functionally graded plates. Applied Mathematical Modelling, 30(1), 67-84.

[30] Zenkour, A.M. (2004) Buckling of fiber-reinforced viscoelastic composite plates using various plate theories. Journal of Engineering Mathematics, 50(1), 75-93.

[31] Zenkour, A.M. (2004) Thermal effects on the bending response of fiber-reinforced viscoelastic composite plates using a sinusoidal shear deformation theory. Acta Mechanica, 171(3-4), 171-187.

[32] Javaheri, R. and Eslami, M.R. (2002) Thermal buckling of functionally graded plates based on higher order theory. Journal of Thermal Stresses, 25(7), 603-625. 